\title{
The tales of two organic cation transporters, OCT-1 and OCT-2, in Caenorhabditis elegans
}

\section{Dindial Ramotar}

Maisonneuve-Rosemont Hospital Research Center, and the Université de Montréal, Faculty of Medicine, Department of Medicine, Montréal, Quebec, Canada.

*Corresponding Author: E-mail: dindial.ramotar@umontreal.ca; Tel.: (514) 252-3400 ext. 4684; Fax: (514) 252-3430

Received: May 25, 2017; Revised: July 13, 2017; Published: September 29, 2017

\begin{abstract}
Solute carrier transporters, previously thought to perform roles in the transport of ions and various nutrients are now assigned broader functions. These transporters have recently been shown to permit entry of therapeutic drugs into cells. There is growing interest to understand the broad spectrum of drugs and chemical compounds that are recognized by these transporters such that specific ligands can be used as therapeutics to target definite physiological pathways. To facilitate this investigation, simpler and cost effective model systems are needed, one of such is the live whole model animal Caenorhabditis elegans (C. elegans) that offers a multitude of advantages. In general, studies with $C$. elegans are feasible due to the simplicity of the readouts that include lifespan, brood size, germ cell death, and visualization by epifluorescent microscopy, which can be set up in any laboratory. In C. elegans, two solute carrier transporters, the organic cation transporters OCT-1 and its paralogue OCT-2 have been partially characterized. OCT-1 mutants display a significantly reduced lifespan and brood size, as well as exhibiting an increased susceptibility towards oxidative stress and a subset of DNA damaging agents. These multiple phenotypes are directly linked to OCT-1 depletion causing upregulation of OCT-2, as RNAi-mediated downregulation of OCT-2 rescues the oct-1 mutant phenotypes. Thus, in C. elegans OCT-1 exerts control onto OCT-2, and this latter transporter plays a predominant role in the uptake of various ligands. We first showed that OCT-2 can efficiently mediate uptake of the widely used anticancer drug doxorubicin into the animals, but prevented uptake upon its downregulation. Additional ligands of OCT-2 including cisplatin and camptothecin were revealed by ligand-docking prediction studies. These analyses generated docking scores indicating that OCT-2 can make robust contact with a number of therapeutics and anticancer drugs, as well as chemical compounds that possess the ability to target specific physiological pathways. Several of the compounds displaying high docking scores with OCT-2 were validated and indeed found to be substrates that OCT-2 transported into the animals. This review provides an insight how the transporters OCT-1 and OCT-2 of a simple model organism C. elegans can be exploited to report on the cytotoxicities and genotoxicities of therapeutic agents, as well as trace amounts of undocumented toxic compounds with neoplastic potentials that are present in the environment.
\end{abstract}

\section{Keywords}

drug uptake and resistance; $C$. elegans organic cation transporters; germ cells apoptosis; DNA damage and repair pathways

\section{Introduction}

The mainstay chemotherapeutics such as anthracyclines, cisplatin and radiomimetic drugs are often used to eliminate cancer cells and reduce the burden of tumors by inflicting damages to the genome [1-3]. These genotoxic agents are administered to cancer patients in combination, and in some cases with small 
molecule inhibitors to block DNA repair pathways with the aim of sensitizing cancer cells towards the chemotherapeutic regimens [4-8]. Although these molecules are hydrophilic in nature, it was commonly accepted that they diffuse into cells. This notion has been challenged as most chemotherapeutics are charged molecules and structurally unrelated, and thus cannot easily cross the plasma membrane barrier. Moreover, a single chemotherapeutic agent does not show the same genotoxicity when given to different cell types, as would be expected in the case of simple drug diffusion. In fact, there is now growing evidence that these charged anticancer drugs depend on facilitated transport mechanisms to enter cells in order to effectively reach the target sites.

In human cells, there are over 450 solute carrier transporters, which are believed to facilitate the uptake of nutrients and ions. However, these transporters also perform functions that are distinct as defects in $30 \%$ of the transporters are linked to diseases that may be related to imbalances in the uptake of nutrients and ions at specific locations. For example, mutations in SLC22A4 lead to Crohn disease, which affects the gastrointestinal tract and mutations in SLC12A3 lead to Gitelman's syndrome characterized by low blood pressure $[9,10]$. Besides the uptake of nutrients and ions, some of these transporters are actively involved in the influx of drugs for treating various illnesses. At least 12 of the transporters are specifically involved in the transport of drugs approved by the Food and Drug Administration (FDA), e.g, SLC6A3 transports methylphenidate used for treating psychiatric disorders [11]. In addition, a few of the solute carriers have been shown to mediate uptake of anticancer drugs. For example, the SLC35F2 transporter is completely responsible for the uptake into human tumour cells of the anticancer drug YM155, which is currently in clinical evaluation [12]. Another recent study established that the organic cation transporter SLC22A2 (OCT2) is required for oxaliplatin uptake into renal cells and that downregulation of OCT2 is a cause for oxaliplatin resistance in renal cell carcinoma [13]. However, these resistant renal cell carcinoma can be sensitized to oxaliplatin upon restoring OCT2 expression [13]. In a final example, the functional level of another member of the organic cation transporter family, SLC22A1 (OCT1), was shown to be a critical marker for the responses of chronic-phase chronic myeloid leukemia towards the tyrosine kinase inhibitor imatinib [14]. Patients with low activity levels of OCT1 respond poorly to imatinib and exhibit lower overall survival as compared to those with high levels of OCT1 [14]. This same transporter was also shown to transport the diabetes drug metformin, which decreases production of hepatic glucose, thus underscoring the possibility that many of the transporters may likely recognize multiple substrates [15]. These foregoing studies raise the possibility that although the transporters may have evolved to mediate uptake of common nutrients and ions, they have the ability to recognize distinct ligands. While it is difficult to determine the ligands for each transporter, it is anticipated that with a given substrate the transporter can be identified through biochemical and genetic analyses.

Since anthracyclines are widely used chemotherapeutic agents and initially believed to diffuse into cells, there has been little interest to determine whether a transporter exists for these drugs. We challenged the initial view and used anthracyclines as the ligands to search for a transporter. Below highlights the key findings that establish the existence of a human transporter OCT1, which mediates uptake of anthracyclines into cells and how this discovery was exploited to uncover similar transporters in the model organism Caenorhabditis elegans (C. elegans). 


\section{Human OCT1 transports anthracyclines}

Anthracyclines such as doxorubicin and daunorubicin act by intercalating with the DNA and blocking the function of DNA topoisomerase leading to the accumulation of DNA lesions and subsequent cell death [16]. Monitoring anthracyclines entry into cells was made possible as these drugs autofluoresce at $640 \mathrm{~nm}$, which provides an important physical property for the development of in vitro assays to quantitatively assess the intracellular uptake levels. These in vitro assays monitor drug uptake into the cells as a function of time using quantitative approaches such as fluorescence-activated cell sorting (FACS) analysis and fluorometry, as well as a semi-quantitative method involving epifluorescence microscopy [17, 18]. Using these assays and a panel of cancer cell lines such as the leukemia HL60 and the ovarian TOV222G2 cell lines, it was shown that daunorubicin or doxorubicin at $5 \mu \mathrm{M}$ enters these cells in a time- and a concentration-dependent manner and reaching saturation within $60 \mathrm{~min}$ [19]. These observations establish for the first time that there is an active transport mechanism for anthracyclines into cells, eliminating the possibility that diffusion accounts for the major accumulation of the drug into cells [19]. Although earlier studies claimed that the active transport mechanism for doxorubicin uptake involved the human SLC22A16 (hCT2/OCT6) and SLC22A4 (OCTN1) transporters, subsequent findings proved that these were not the transporters [18, 20,21]. However, it was studies from the budding yeast Saccharomyces cerevisiae that provided clues to the actual transporter by directly linking anthracycline-mediated uptake to the family of OCT transporters. In human cells, there are three OCT homologues OCT1, OCT2 and OCT3 that are members of one family of organic cation transporters all of which can transport choline with varying affinities. We therefore conducted competition experiments with the human cancer cell lines and documented that choline completely blocked the uptake of daunorubicin into the cells when added at nearly equimolar amounts [19]. Since OCT1 has a higher affinity for choline than OCT2 and OCT3 and can mediate uptake of several different ligands into cells, it is strongly suggested that it could be a suitable candidate to explore for a role in anthracycline uptake. The first direct evidence came from silencing OCT1 expression by shRNA in the ovarian cancer cells TOV222G2, which sharply reduced the OCT1 protein level, as revealed by anti-OCT1 antibodies [19]. These OCT1 downregulated cells showed nearly $85 \%$ reduction in daunorubicin uptake when compared to the control shRNA that showed no effect [19]. As a corollary experiment, overproduction of OCT1 which is localized to the plasma membrane, stimulated daunorubicin uptake into the cancer cell lines [19]. Collectively, these studies provide the first convincing evidence that OCT1 functional level might be pivotal in determining the effectiveness of treatment with anthracyclines. Since anthracyclines are used for treating leukemia as well as several other types of cancers such as lymphomas, breast, ovarian, and gastric, it is anticipated that patients who are refractory to anthracycline treatment are likely to be defective in the uptake transporter [22-26]. Conversely, patients with induced levels or activated OCT1 could be hypersensitive to anthracyclines.

Because the human OCT1 transporter displays broad substrate specificity, it is imperative to investigate whether its function in drug transport can be influenced by the presence of additional drugs, nutrients, and or the status of other influx and efflux transporters. As such, it seems logical that a well characterized OCT1 transporter would be beneficial in choosing the appropriate drug, without competition by other substrates, for targeted entry into cells. To rapidly address these issues, it would require a simple live whole model system that is readily tenable to genetic modifications and cost effectiveness such as Caenorhabditis elegans. 


\section{C. elegans as a model to facilitate studies on drug uptake transporters}

C. elegans has been chosen as the whole model system as it offers a multitude of advantages over mammalian cells to rapidly study many highly conserved biological processes [27]. C. elegans developed from eggs to the adult stage within three days spanning four larval stages. Genes can be readily downregulated by simply feeding the animals with bacteria expressing double stranded RNA from a segment of the coding region of the targeted gene. C. elegans are transparent and facilitate easy visualization of germ and somatic cells by microscopy. During the last decade, $C$. elegans have been instrumental in several drug discovery programs that include the search for new antifungals and compounds to limit oxidative stress $[27,28]$, although the contributions of uptake transporters in these studies were not considered [29]. Therefore, it is unclear how C. elegans uptake transporters would have influenced many of the high-throughput screens performed so far to identify novel small molecules that act, for example, as antimicrobials. One possibility is that the yield of bioactive compounds typically recovered from these screens, ranging from 0.03 to $1 \%$, could be substantially higher if there is greater influx of the molecules into the animal cells via elevated expression of uptake transporters [27, 30]. Thus, defining the function and substrate specificities of uptake transporters in C. elegans will be advantageous towards improving the strategies employed to identify novel bioactive molecules. Highlighted below are the current progresses regarding the roles of a previously and a recently discovered organic cation transporter, OCT-1 and OCT-2, respectively, in C. elegans and how these transporters can be exploited for drug testing.

\section{Uptake transporters in C. elegans}

In comparison to the human genome, there are far fewer organic cation transporters in C. elegans following an analysis of the sequence information in the Wormbase. These $C$. elegans transporters include the partially characterized OCT-1 and OCT-2 and at least six other putative transmembrane transporters PES-23, OAT-1, SVOP-1, STR-176, HMT-1.1/1.2 and GEM-1. Far less is known about these latter six putative transporters and will not be discussed further. The C. elegans OCT-1 and OCT-2 are counterparts of the human OCTs and little is still known about their roles and affinities towards distinct ligands. Both OCT-1 and OCT-2 share $22.56 \%$ identity at the amino acid level, but OCT-2 differs from OCT-1 as it harbours an extra 172 amino acid residues at the N-terminal, which has no homology to OCT-1.

In 1999, OCT-1 was the first uptake transporter identified from C. elegans [31]. The oct-1 gene was designed to be expressed in mammalian cells and shown to mediate the transport of the organic cation tetraethylammonium, a prototypical substrate used for classifying OCTs, suggesting that OCT-1 has the ability to function as an uptake transporter [31]. At the time, no extensive study was done to assess the detail substrate specificity of OCT-1 following its expression in human cells. It was much later when the next study appeared in 2013 showing that $C$. elegans deleted for the oct-1 gene exhibited a shortened lifespan, as well as an increased susceptibility to oxidative stress [29]. These observations led to the proposition that OCT-1 facilitates the import of antioxidants into the animals, which is required to protect oct-1 mutant animals from oxidative stress [29]. However, the level of the purported substrate of OCT-1, the antioxidant ergothioneine, was not altered in oct-1 mutant animals as compared to the parent [29]. Therefore, it seems plausible that an alternative explanation could account for the oct-1 mutant animal phenotypes.

In an attempt to find substrates for OCT-1, we expressed the $C$. elegans oct-1 gene in another heterologous model system, namely the budding yeast Saccharomyces cerevisiae. Since human OCT1 can 
transport anthracyclines, we first tested these chemotherapeutic drugs. We observed that expression of $C$. elegans OCT-1 can trigger the uptake of doxorubicin into yeast cells. The yeast strain we used was defective in Agp2, which has been reported to be a plasma membrane amino acid transporter that also performs a regulatory role [32]. Deletion of the AGP2 gene in yeast blocked the expression of several target genes including the polyamine transporters Dur3 and Sam3 [18]. Both Dur3 and Sam3 can independently transport doxorubicin when overexpressed in yeast cells lacking Agp2 [18]. Therefore, it is not clear whether the expression of $C$. elegans OCT-1 in yeast substituted for the regulatory function of Agp2 or directly for the roles of Dur3 and Sam3, since these transporters also mediate uptake of doxorubicin [18]. As such, it became necessary to test directly whether OCT-1 might mediate the transport of doxorubicin into $C$. elegans. However, the existence of another related member of the organic cation transporter family, namely OCT-2, in C. elegans, suggests that there needs to be a strategy to test the contribution of each transporter towards doxorubicin uptake. Prior to our work, there was no function assigned to this putative transporter OCT-2 [33]. Moreover, there are no animals in the consortiums, USA or Japan, carrying either partial or complete deletion of the oct-2 gene, as in the case of oct-1. Thus, to examine whether OCT-1 or OCT-2 or both have roles in the uptake of doxorubicin in C. elegans, we used RNA-interference (RNAi) feeding bacteria to generate animals with different genotypes, in particular, either downregulated for one or both transporters [33].

Using this approach, we uncovered several novel findings regarding the OCT-1 and OCT-2 transporters in C. elegans. First, unlike the oct-1 null animals, downregulation of oct-2 did not affect the lifespan of the animals. Second, perhaps most interesting, the depletion of OCT-2 rescued the shortened lifespan of animals already deleted for the oct-1 gene [33]. This striking observation prompted the possibility that OCT-1 might influence OCT-2 function, whereby oct-1 downregulation leads to oct-2 upregulation. This notion is based on the fact that OCT1 knockout mice manifest upregulation of two related transporter genes, OCT2 and OCT3 [34]. And third, quantitative PCR analysis indeed revealed that downregulation of oct-1 led to the upregulation of oct-2 expression in several tissues of the animals, while several control genes, such as act-1 encoding actin, were unaffected [33]. As such, we proposed that OCT-2 upregulation could be responsible for triggering uptake of toxic environmental compounds into the animals and thus account for the shortened lifespan displayed by the oct-1 animals.

There is evidence to support that the upregulation of OCT-2 leads to the import of toxic compounds from the environment into the animals. This was documented using a simple reporter animal that harbours within its genome the oxidative stress response gene gst- 4 encoding glutathione $S$-transferase 4 fused to the Green Fluorescent Protein (GFP) $[35,36]$. In C. elegans SKN-1, the counterpart of the mammalian NRF2, senses oxidative stress and turn on the expression of gst-4. We used the gst::gfp reporter animals to monitor oxidative stress caused by toxic compounds and showed that these animals expressed a basal level of GST-4::GFP in the intestine. However, the expression level of the GST-4::GFP was greatly stimulated following RNAi downregulation of oct-1 that led to the upregulation of oct-2 [33]. Consistent with this observation, downregulation of the oct-2 transporter gene by RNAi lowered the basal levels of GST-4::GFP expression in the reporter strain [33]. Thus, oct-2 upregulation, via oct-1 downregulation, allows entry of toxic compounds such as prooxidants into $C$. elegans. These prooxidants increased the oxidative stress of the animals that could cause oxidative damages to various macromolecules, such as DNA, and leading to the shortened lifespan of the oct-1 null animals. 


\section{OCT-2 mediates the accumulation of doxorubicin in C. elegans tissues}

Equipped with the new knowledge on the regulation of oct-2 by OCT-1, we checked if these transporters possess the ability to drive doxorubicin uptake and accumulation into $C$. elegans tissues. In the design of the experiment, the fluorescent property of doxorubicin, emitting fluorescence at wavelengths of $\lambda^{\mathrm{ex}} 470 \mathrm{~nm}-\lambda^{\mathrm{em}} 585 \mathrm{~nm}$, became a convenient means to monitor its uptake in situ by imaging the pharynx, a very large and visible organ. On the basis of the previous studies in both human and yeast cells, we anticipated that $C$. elegans OCT-1 would act as an uptake transporter and that oct-1 null animals would display very little doxorubicin uptake $[18,19]$. Unexpectedly, we observed that the $C$. elegans oct-1 null mutant exposed to doxorubicin depicted a markedly stimulated fluorescence intensity of the drug in the pharynx, as compared to the parent animals [33]. The simplest explanation for this finding is that deletion of the oct-1 gene resulted in the upregulation of oct-2, and it is OCT-2 that is responsible for doxorubicin uptake. Indeed, the downregulation of oct-2 via RNAi feeding bacteria of the oct-1 null mutant completely blocked the uptake of doxorubicin, clearly indicating that OCT-2 and not OCT-1 has a predominant role in the drug uptake [33]. These observations in C. elegans contradict the previously reported role for OCT-1 as an uptake transporter $[18,31]$. It is possible that in the natural environment OCT-1 may be modified to exert a distinct role other than functioning as an uptake transporter or alternatively exhibits specificity for an entirely different class of substrates. Based on the present evidence, we believe that OCT-2 might be the key transporter that mediates doxorubicin uptake into C. elegans. OCT2 also has the ability to recognize and transport other cationic compounds. For example, choline can compete for the uptake of doxorubicin entry into $C$. elegans, suggesting that it is likely a substrate for OCT$2[33,37]$. In fact, choline is a substrate for human OCT1 and it impedes the uptake of daunorubicin into human cell lines [19]. It is reasonable to propose that any compound that competes for doxorubicin uptake into the $C$. elegans oct-1 null animals is likely a putative substrate for OCT-2, thus providing a foundation to develop a screening tool to find therapeutic compounds that are transported by OCT-2. In developing this assay further, it is noteworthy that other factors should be considered such as compounds, e.g., spermine that could quench the fluorescence of doxorubicin leading to false positives. Nonetheless, the same assay can be extended to find compounds that could stimulate doxorubicin uptake revealing activators for OCT-2.

\section{OCT-1 and OCT-2 play a pivotal role in controlling germ cell death}

In a manner similar to many stem cell systems, C. elegans has a self-renewing germ cell population [38]. These germ cells are very sensitive to genotoxic compounds and respond by using conserved DNA repair mechanisms to faithfully maintain genomic stability [39]. Germ cells with extensive DNA damage undergo apoptosis and cannot form viable embryos, and which can be quantified in vivo by examining for corpses in the proximal zone of the gonad arm [38, 40,41]. These corpses are easily seen by staining with the DNA dye acridine orange [38, 40,41]. Thus, germ cell apoptosis is a suitable in vivo reporter system to determine whether natural compounds and those in the growth environment of the animals are genotoxic. We took advantage of this reporter system to assess whether OCT-1 and OCT- 2 control the extent of germ cell death triggered by genotoxic agents, in particular, those compounds that cannot be tracked directly for their uptake kinetics due to the absence of a fluorescence signal or the availability of radioactively labeled form. In the wild type animals grown under normal conditions, there are typically two to four germ cell corpses [42]. However, when oct-1 is downregulated the animals depicted an average of five to eight apoptotic cells, perhaps resulting from an increase import of genotoxic prooxidants by the elevated levels of the OCT-2 transporter leading to dead embryos [33]. Interestingly, downregulation of oct-2 in the oct-1 null mutant reduced the germ cell death, and in some animals to undetectable level. It seems that OCT-2 
possesses the ability to recognize and transport toxic compounds from the growth environment into the animals which then induced germ cell death. At least, these toxic compounds can turn on the oxidative stress reporter GST-4::GFP, suggesting that they compose of prooxidants with the ability to cause genotoxicity in various tissues [33]. In fact, the oct-1 deficient animals produce an elevated level of dead embryos, which are believed to be the result of the accumulation of genotoxic DNA lesions under normal growth conditions, and therefore account for the reduced lifespan observed in these animals.

Since doxorubicin acts by damaging the DNA and previously shown to trigger germ cell apoptosis in $C$. elegans [43], then modulating OCT-2 level should determine the extent of doxorubicin-induced germ cell death. Indeed, oct-1 deletion animals showed substantial levels of germ cell deaths when treated with doxorubicin, as opposed to the wild-type. This phenomenon was greatly diminished following OCT-2 downregulation in the doxorubicin treated oct-1 null animals. These observations are consistent with the model that oct-1 deletion led to OCT-2 upregulation, which then mediates doxorubicin uptake that induces germ cell death. Therefore, germ cell death is a genuine reporter for OCT-2 dependent uptake of genotoxic agents.

\section{Potential substrates of OCT-2}

While it is clear that additional substrates will be recognized by OCT-2, it is an arduous task to test each potential substrate, for example, by competing for doxorubicin uptake. As such, we undertook an in silico approach by first making predictions of the structure of the transporter using the I-TASSER protein structure prediction server [44]. The analysis predicted 3D structures not only for OCT-2, but also OCT-1, revealing that both possessed the entire 12 transmembrane domain helices and belonging to the solute carrier transporter family [33, 45]. The predicted 3D structures of the transporters were then modeled with drugs to look for interactions using the BSP-SLIM and $\mathrm{COACH}$ algorithms. These programs approximate the amino acid residues of the transporters constituting the ligand-protein docking sites and thereby resulting in a docking score $[46,47]$. A number of drugs were analyzed and these were mostly DNA damaging agents including cisplatin and camptothecin and small molecules with the ability to inhibit DNA repair such as olaparib and methoxyamine. Of the 19 potential substrates, four resulted with a docking score of zero, whereas the other 15 revealed docking scores > 3.5 [33]. Many showed higher docking scores with OCT-2 than OCT-1, favoring robust binding to OCT-2 [33]. From the molecules tested, it seems that OCT-2 may distinguish specific substrates to be transported into $C$. elegans tissues by recognizing an overall positive charge and perhaps structural features that include aromatic groups. It is noteworthy that there are compounds that can damage the DNA and induce apoptotic corpses such as melphalan and methoxyamine. However, these agents do not dock onto OCT-2 or OCT-1, and may therefore require alternative transporters to enter the animals [33]. It is anticipated that protein-ligand modeling studies should reveal novel substrates for these uptake transporters, but these ligands must be experimentally validated as described below for the anticancer drug cisplatin and an inhibitor of DNA repair.

\section{OCT-2-dependent transport of cisplatin stimulates germ cell death in C. elegans mutants defective in DNA repair}

Cisplatin, a predicted ligand of OCT-2, is a member of the platinum family of anticancer drugs that damages the DNA by creating intra- and inter-strand DNA cross-links [48]. These DNA lesions are repaired by the nucleotide excision DNA repair pathway, which has the ability to recognize helix distortion followed 
by incisions on either side of the lesion [49]. Thus, defects in the nucleotide excision repair pathway cause hypersensitivity to cisplatin [49]. As cisplatin is not available in labeled forms, but can be monitored by liquid chromatography mass spectrometry, the germ cell apoptotic assay provides a remarkable alternative reporter for the uptake of the drug $[39,50]$. The analysis revealed that cisplatin induced an increase level of germ cell death in the wild type animal, and which was further stimulated in the oct-1 null mutants [33]. Downregulation of oct-2 in the oct-1 null mutants prevented the cisplatin-induced germ cell death indicating that OCT-2 plays a central role in mediating the genotoxic effects of cisplatin [33]. Thus, the ligand-docking studies described above serve as a useful prediction platform to screen for OCT-2 substrates.

One prediction from these findings is that the elevated uptake of genotoxic agents into animals defective in DNA repair should result in lethal phenotypes. There are several mutants in the $C$. elegans consortium carrying defects in the major DNA repair pathways and which are suitable to test whether OCT2 upregulation can trigger sensitization of the animals to DNA damage-induced germ cell death. For example, the xpa-1 deletion mutant lacking the XPA-1 protein needed for nucleotide excision repair of bulky DNA lesions exhibited enhance germ cell death when exposed to cisplatin following downregulation of oct-1. In contrast, depletion of oct-2 by RNAi in the xpa-1 mutant animals suppressed the high level of apoptotic cells observed in this mutant upon exposure to cisplatin. These data indicate that upregulation of oct-2 burdens the xpa-1 mutant animals with cisplatin-induced DNA lesions leading to the enhancement of germ cell death [51] [52]. Thus, the current studies suggest that by combining defects of various DNA repair pathways with functional organic cation transporters such as OCT-2, it is possible to determine whether an unknown compound has genotoxic effects and the type of lesions it may create.

\section{OCT-2 transports the RAD51 inhibitor B02}

In addition to the genotoxic agents that docked onto OCT-2, the analyses revealed that many chemical compounds such as 3-benzyl-2-[(E)-2-pyridin-3-ylethenyl]quinazolin-4-one (B02, CID: 5738263), referred to as B02, has the ability to robustly interact with OCT-2, and not OCT-1. In human cells, B02 has been shown to interfere with the DNA strand exchange and the nuclear foci formation catalyzed by the DNA repair protein RAD51. RAD51 is required to repair DNA double strand breaks during meiosis and in response to DNA damaging agents [53-55]. Examination of the pharmacological effect of B02 in C. elegans revealed that this inhibitor caused sterility in the oct-1 null mutant. This is marked by the production of inviable embryos and a decrease in the number of viable animals, phenotypes that mimic those of the rad-51 homozygotes [56]. As predicted from the model, downregulation of oct- 2 in the oct-1 null mutant prevented the B02 ligand from causing sterility [33]. Under these conditions, the oct-1 null mutant produced viable embryos that resulted in nearly normal levels of viable animals [33]. From these data, it is clear that B02 can also inhibit RAD-51 function in C. elegans and this depends on the function of the OCT-2 uptake transporter [33]. Thus, the docking scores of ligands and proper readouts from the targeted pathway provide valuable tools to monitor transporter-mediated drug uptake into $C$. elegans.

\section{Perspectives}

There is no evidence yet that OCT-1 can function as an uptake transporter in C. elegans, although when expressed in two different heterologous model system, human and yeast cells, it can stimulate uptake of tetraethylammonia and doxorubicin, respectively $[18,31]$. While in human cells downregulation of OCT1 caused resistance to anthracyclines, this was not the case in $C$. elegans devoid of OCT-1, thus a direct role for OCT-1 in the uptake of anthracyclines can be excluded. Whether OCT-1 acts as an uptake transporter 
for selective drugs remains a possibility. Nonetheless, in C. elegans OCT-1 has a role in exerting control on oct-2 expression, and it is OCT-2 that is primarily involved in the uptake of doxorubicin and other substrates. We believe that OCT-2 has a broad specificity and can transport various substrates, including toxic environmental compounds, from the pharynx into the intestine (Fig. 1). The OCT-2 in the intestine then transports the substrates into the vicinity of the oocytes and embryos, which are also equipped with the OCT-2 transporter (Fig. 1). Thus, removal of OCT-2 stands to protect the animal from germ cell death induced by environmental and chemical toxicants. The data obtained so far underscore the importance of uptake transporters in regulating the entry of drugs into cells and raise the possibility that (i) the drugresistance and -sensitivity exhibited by cancer patients could be governed at the level of the uptake transporters and (ii) the absence of one transporter could greatly impact the function of another.

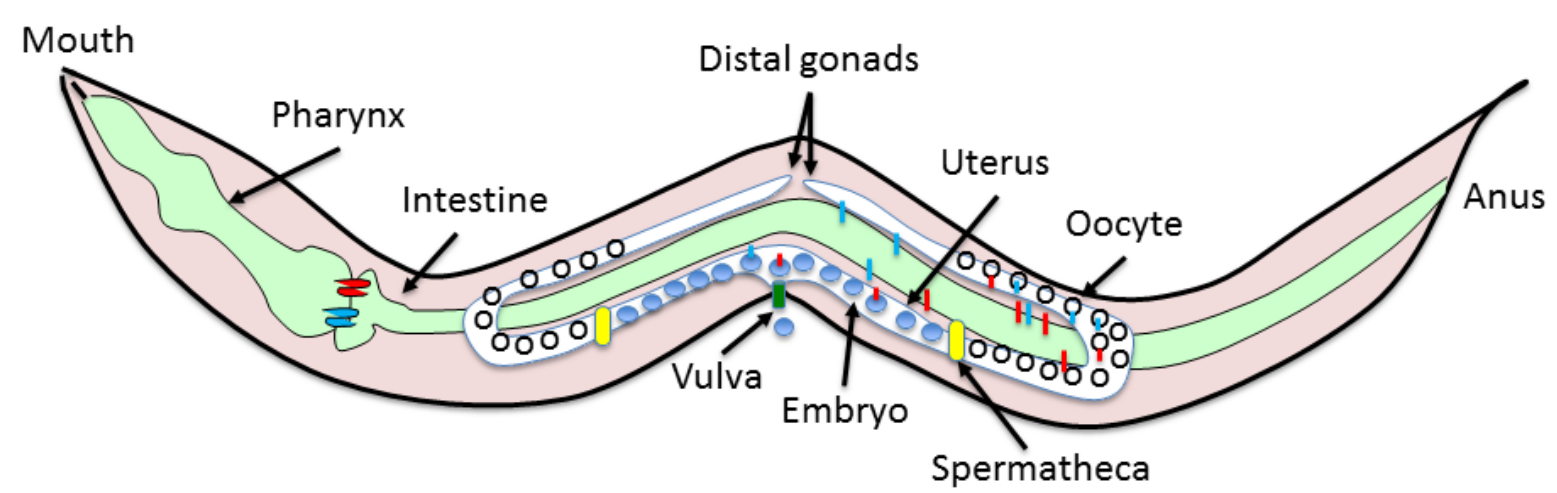

Figure 1. Illustration of key features of an adult $C$. elegans hermaphrodite. The blue squiggly line represents the putative location of OCT-1 transporter, while the red squiggly line and short vertical red bar represent the putative locations of OCT-2 transporter on the pharynx, intestine, oocytes and embryos.

The studies to date also raised additional concern and that is whether the upregulated OCT-2 transporter can import compounds from the growth media that cause DNA damage and ultimately leading to genomic instability over time. If this were the case, then individuals with deregulated uptake transporters are likely at increased risk of being bombarded with genotoxic compounds that could lead to various diseases such as cancers. In fact, preliminary data revealed that the upregulation of OCT-2, via RNAi downregulation of oct-1, resulted in at least a two-fold increase in spontaneous mutations in a $C$. elegans strain designed to monitor frame shift mutations at the lacZ reporter gene [57].

The downregulation of oct-1 leading to the upregulation of oct-2 was an unexpected finding, and provides a compelling argument that the animal has evolved tight regulation of OCT-2 presumably to control uptake of unwanted compounds. OCT-1 could perform a function as a surface sensor that acts as non-transporting transceptors by sensing the availability of nutrients and signaling the regulation of downstream plasma membrane transporters $[32,58]$. Thus, when nutrients are scarce, OCT-1 might serve as a sensor to promote the upregulation of OCT-2 to scavenge the limited resources, but sustain basal expression of OCT-2 when nutrients are in surplus. This mode of regulation has been characterized in $S$. cerevisiae, Drosophila melanogaster and Homo sapiens [59-61]. Thus, in view of the increasing number of sensors that are currently being identified in various organisms, it is plausible that $C$. elegans OCT-1 may indeed act either as a non-transporting or transporting sensor leading to the regulation of oct-2 expression. If such a model is possible, other factors including kinases and transcriptional activators would be involved in conveying the signal from OCT-1 to promote OCT-2 expression as shown for the S. cerevisiae Ssy1 amino acid sensing pathway [62].

We propose that exploiting OCT-2 in C. elegans could have far reaching applications that supersede 
other whole model systems in drug discovery programs with respect to cost and time. Thus, maintaining the OCT-2 transporter at optimal levels should be a useful step to incorporate into any high-throughput screens to improve the efficiency of identifying cationic bioactive molecules from chemical libraries. The overexpressed OCT-2 is expected to significantly alter its kinetics such that it can transport chemicals that are present at lower concentrations in the libraries. We strongly suggest that the previous barriers posed by $C$. elegans to find bioactive molecules could be explained by the lack of an activated mechanism to efficiently take up compounds at lower concentrations.

\section{Acknowledgments}

Special thanks to Taramatti Harihar for her editorial comments. This work was funded by the research project grant 201610PJT (377317) to D.R. from the Canadian Institute of Health Research.

\section{References}

[1] A.C. Begg, F.A. Stewart, C. Vens. Strategies to improve radiotherapy with targeted drugs, Nat Rev Cancer 11 (2011) 239-253.

[2] C.J. Lord, A. Ashworth. The DNA damage response and cancer therapy, Nature 481 (2012) 287-294.

[3] K. Cheung-Ong, G. Giaever, C. Nislow. DNA-damaging agents in cancer chemotherapy: serendipity and chemical biology, Chem Biol 20 (2013) 648-659.

[4] M.R. Kelley, D. Logsdon, M.L. Fishel. Targeting DNA repair pathways for cancer treatment: what's new?, Future Oncol 10 (2014) 1215-1237.

[5] W.G. Kaelin, Jr. Synthetic lethality: a framework for the development of wiser cancer therapeutics, Genome Med 1 (2009) 99.

[6] A. Bapat, M.L. Fishel, M.R. Kelley. Going ape as an approach to cancer therapeutics, Antioxid Redox Signal 11 (2009) 651-668.

[7] S.P. Jackson, J. Bartek. The DNA-damage response in human biology and disease, Nature 461 (2009) 1071-1078.

[8] A. Ciccia, S.J. Elledge. The DNA damage response: making it safe to play with knives, Mol Cell 40 (2010) 179-204.

[9] V.D. Peltekova, R.F. Wintle, L.A. Rubin, C.I. Amos, Q. Huang, X. Gu, B. Newman, M. Van Oene, D. Cescon, G. Greenberg, A.M. Griffiths, P.H. St George-Hyslop, K.A. Siminovitch. Functional variants of OCTN cation transporter genes are associated with Crohn disease, Nat Genet 36 (2004) 471-475.

[10] S.T.D. Consortium, A.L. Williams, S.B. Jacobs, H. Moreno-Macias, A. Huerta-Chagoya, C. Churchhouse, C. Marquez-Luna, H. Garcia-Ortiz, M.J. Gomez-Vazquez, N.P. Burtt, C.A. AguilarSalinas, C. Gonzalez-Villalpando, J.C. Florez, L. Orozco, C.A. Haiman, T. Tusie-Luna, D. Altshuler. Sequence variants in SLC16A11 are a common risk factor for type 2 diabetes in Mexico, Nature 506 (2014) 97-101.

[11] A. Cesar-Razquin, B. Snijder, T. Frappier-Brinton, R. Isserlin, G. Gyimesi, X. Bai, R.A. Reithmeier, D. Hepworth, M.A. Hediger, A.M. Edwards, G. Superti-Furga. A Call for Systematic Research on Solute Carriers, Cell 162 (2015) 478-487.

[12] G.E. Winter, B. Radic, C. Mayor-Ruiz, V.A. Blomen, C. Trefzer, R.K. Kandasamy, K.V. Huber, M. Gridling, D. Chen, T. Klampfl, R. Kralovics, S. Kubicek, O. Fernandez-Capetillo, T.R. Brummelkamp, G. Superti-Furga. The solute carrier SLC35F2 enables YM155-mediated DNA damage toxicity, Nat Chem Biol 10 (2014) 768-773.

[13] Y. Liu, X. Zheng, Q. Yu, H. Wang, F. Tan, Q. Zhu, L. Yuan, H. Jiang, L. Yu, S. Zeng. Epigenetic activation of the drug transporter OCT2 sensitizes renal cell carcinoma to oxaliplatin, Sci Trans/ Med 8 (2016) 348 ra397. 
[14] D.L. White, V.A. Saunders, P. Dang, J. Engler, T.P. Hughes. OCT-1 activity measurement provides a superior imatinib response predictor than screening for single-nucleotide polymorphisms of OCT-1, Leukemia 24 (2010) 1962-1965.

[15] M.L. Becker, L.E. Visser, R.H. van Schaik, A. Hofman, A.G. Uitterlinden, B.H. Stricker. Genetic variation in the organic cation transporter 1 is associated with metformin response in patients with diabetes mellitus, Pharmacogenomics $J 9$ (2009) 242-247.

[16] T.J. Westmoreland, S.M. Wickramasekara, A.Y. Guo, A.L. Selim, T.S. Winsor, A.L. Greenleaf, K.L. Blackwell, J.A. Olson, Jr., J.R. Marks, C.B. Bennett. Comparative genome-wide screening identifies a conserved doxorubicin repair network that is diploid specific in Saccharomyces cerevisiae, PLoS One 4 (2009) e5830.

[17] K.K. Karukstis, E.H. Thompson, J.A. Whiles, R.J. Rosenfeld. Deciphering the fluorescence signature of daunomycin and doxorubicin, Biophys Chem 73 (1998) 249-263.

[18] N. Brosseau, E. Andreev, D. Ramotar. Complementation of the Yeast Model System Reveals that Caenorhabditis elegans OCT-1 Is a Functional Transporter of Anthracyclines, PLoS One 10 (2015) e0133182.

[19] E. Andreev, N. Brosseau, E. Carmona, A.M. Mes-Masson, D. Ramotar. The human organic cation transporter OCT1 mediates high affinity uptake of the anticancer drug daunorubicin, Sci Rep 6 (2016) 20508.

[20] M. Okabe, M. Unno, H. Harigae, M. Kaku, Y. Okitsu, T. Sasaki, T. Mizoi, K. Shiiba, H. Takanaga, T. Terasaki, S. Matsuno, I. Sasaki, S. Ito, T. Abe. Characterization of the organic cation transporter SLC22A16: a doxorubicin importer, Biochem Biophys Res Commun 333 (2005) 754-762.

[21] M. Okabe, G. Szakacs, M.A. Reimers, T. Suzuki, M.D. Hall, T. Abe, J.N. Weinstein, M.M. Gottesman. Profiling SLCO and SLC22 genes in the $\mathrm{NCl}-60$ cancer cell lines to identify drug uptake transporters, Mol Cancer Ther 7 (2008) 3081-3091.

[22] J.W. Lown. Anthracycline and anthraquinone anticancer agents: current status and recent developments, Pharmacol Ther 60 (1993) 185-214.

[23] H. Dohner, E.H. Estey, S. Amadori, F.R. Appelbaum, T. Buchner, A.K. Burnett, H. Dombret, P. Fenaux, D. Grimwade, R.A. Larson, F. Lo-Coco, T. Naoe, D. Niederwieser, G.J. Ossenkoppele, M.A. Sanz, J. Sierra, M.S. Tallman, B. Lowenberg, C.D. Bloomfield. Diagnosis and management of acute myeloid leukemia in adults: recommendations from an international expert panel, on behalf of the European LeukemiaNet, Blood 115 (2010) 453-474.

[24] T.R. Chauncey. Drug resistance mechanisms in acute leukemia, Curr Opin Oncol 13 (2001) 21-26.

[25] J.P. Marie. Drug resistance in hematologic malignancies, Curr Opin Oncol 13 (2001) 463-469.

[26] J.C. Byrd, K. Mrozek, R.K. Dodge, A.J. Carroll, C.G. Edwards, D.C. Arthur, M.J. Pettenati, S.R. Patil, K.W. Rao, M.S. Watson, P.R. Koduru, J.O. Moore, R.M. Stone, R.J. Mayer, E.J. Feldman, F.R. Davey, C.A. Schiffer, R.A. Larson, C.D. Bloomfield. Pretreatment cytogenetic abnormalities are predictive of induction success, cumulative incidence of relapse, and overall survival in adult patients with de novo acute myeloid leukemia: results from Cancer and Leukemia Group B (CALGB 8461), Blood 100 (2002) 4325-4336.

[27] L.P. O'Reilly, C.J. Luke, D.H. Perlmutter, G.A. Silverman, S.C. Pak. C. elegans in high-throughput drug discovery, Adv Drug Deliv Rev 69-70 (2014) 247-253.

[28] T. Kaletta, M.O. Hengartner. Finding function in novel targets: C. elegans as a model organism, Nat Rev Drug Discov 5 (2006) 387-398.

[29] I.K. Cheah, R.L. Ong, J. Gruber, T.S. Yew, L.F. Ng, C.B. Chen, B. Halliwell. Knockout of a putative ergothioneine transporter in Caenorhabditis elegans decreases lifespan and increases susceptibility to oxidative damage, Free Radic Res 47 (2013) 1036-1045.

[30] A.R. Burns, I.M. Wallace, J. Wildenhain, M. Tyers, G. Giaever, G.D. Bader, C. Nislow, S.R. Cutler, P.J. Roy. A predictive model for drug bioaccumulation and bioactivity in Caenorhabditis elegans, Nat Chem Biol 6 (2010) 549-557. 
[31] X. Wu, Y.J. Fei, W. Huang, C. Chancy, F.H. Leibach, V. Ganapathy. Identity of the F52F12.1 gene product in Caenorhabditis elegans as an organic cation transporter, Biochim Biophys Acta 1418 (1999) 239-244.

[32] M. Aouida, M. Rubio-Texeira, J.M. Thevelein, R. Poulin, D. Ramotar. Agp2, a member of the yeast amino acid permease family, positively regulates polyamine transport at the transcriptional level, PLoS One 8 (2013) e65717.

[33] A. Papaluca, D. Ramotar. A novel approach using C. elegans DNA damage-induced apoptosis to characterize the dynamics of uptake transporters for therapeutic drug discoveries, Sci Rep 6 (2016) 36026.

[34] L. Chen, Y. Shu, X. Liang, E.C. Chen, S.W. Yee, A.A. Zur, S. Li, L. Xu, K.R. Keshari, M.J. Lin, H.C. Chien, Y. Zhang, K.M. Morrissey, J. Liu, J. Ostrem, N.S. Younger, J. Kurhanewicz, K.M. Shokat, K. Ashrafi, K.M. Giacomini. OCT1 is a high-capacity thiamine transporter that regulates hepatic steatosis and is a target of metformin, Proc Natl Acad Sci U S A 111 (2014) 9983-9988.

[35] M.L. Fishel, X. Wu, C.M. Devlin, D.P. Logsdon, Y. Jiang, M. Luo, Y. He, Z. Yu, Y. Tong, K.P. Lipking, A. Maitra, N.V. Rajeshkumar, G. Scandura, M.R. Kelley, M. Ivan. Apurinic/apyrimidinic endonuclease/redox factor-1 (APE1/Ref-1) redox function negatively regulates NRF2, J Biol Chem 290 (2015) 3057-3068.

[36] F. Cabreiro, C. Au, K.Y. Leung, N. Vergara-Irigaray, H.M. Cocheme, T. Noori, D. Weinkove, E. Schuster, N.D. Greene, D. Gems. Metformin retards aging in C. elegans by altering microbial folate and methionine metabolism, Cell 153 (2013) 228-239.

[37] K. Hoffmann, F. Grafe, W. Wohlrab, R.H. Neubert, M. Brandsch. Functional characterization of a high-affinity choline transport system in human keratinocytes, J Invest Dermatol 119 (2002) 118121.

[38] A. Gartner, P.R. Boag, T.K. Blackwell. Germline survival and apoptosis, WormBook (2008) 1-20.

[39] H. Lans, W. Vermeulen. Tissue specific response to DNA damage: C. elegans as role model, DNA Repair (Amst) 32 (2015) 141-148.

[40] H.R. Horvitz. Genetic control of programmed cell death in the nematode Caenorhabditis elegans, Cancer Res 59 (1999) 1701s-1706s.

[41] A.L. Craig, S.C. Moser, A.P. Bailly, A. Gartner. Methods for studying the DNA damage response in the Caenorhabdatis elegans germ line, Methods Cell Biol 107 (2012) 321-352.

[42] T.L. Gumienny, E. Lambie, E. Hartwieg, H.R. Horvitz, M.O. Hengartner. Genetic control of programmed cell death in the Caenorhabditis elegans hermaphrodite germline, Development 126 (1999) 1011-1022.

[43] A. Sendoel, S. Maida, X. Zheng, Y. Teo, L. Stergiou, C.A. Rossi, D. Subasic, S.M. Pinto, J.M. Kinchen, M. Shi, S. Boettcher, J.N. Meyer, M.G. Manz, D. Bano, M.O. Hengartner. DEPDC1/LET-99 participates in an evolutionarily conserved pathway for anti-tubulin drug-induced apoptosis, Nat Cell Biol 16 (2014) 812-820.

[44] A. Roy, A. Kucukural, Y. Zhang. I-TASSER: a unified platform for automated protein structure and function prediction, Nat Protoc 5 (2010) 725-738.

[45] L. Sun, X. Zeng, C. Yan, X. Sun, X. Gong, Y. Rao, N. Yan. Crystal structure of a bacterial homologue of glucose transporters GLUT1-4, Nature 490 (2012) 361-366.

[46] H.S. Lee, Y. Zhang. BSP-SLIM: a blind low-resolution ligand-protein docking approach using predicted protein structures, Proteins 80 (2012) 93-110.

[47] J. Yang, A. Roy, Y. Zhang. Protein-ligand binding site recognition using complementary bindingspecific substructure comparison and sequence profile alignment, Bioinformatics 29 (2013) 25882595.

[48] L. Lin, S.W. Yee, R.B. Kim, K.M. Giacomini. SLC transporters as therapeutic targets: emerging opportunities, Nat Rev Drug Discov 14 (2015) 543-560. 
[49] E.C. Friedberg, G.C. Walker, W. Siede, R.D. Wood, R.A. Schultz, T. Ellenberger, DNA Repair and Mutagenesis, ASM Press, Washington, D.C., 2006.

[50] A.N. Shaik, D.A. Altomare, L.J. Lesko, M.N. Trame. Development and validation of a LC-MS/MS assay for quantification of cisplatin in rat plasma and urine, J Chromatogr B Analyt Technol Biomed Life Sci 1046 (2017) 243-249.

[51] X. Yang, J. Fan, A.A. Ishchenko, D. Patel, M.K. Saparbaev, D. Ramotar. Functional characterization of the Caenorhabditis elegans DNA repair enzyme APN-1, DNA Repair (Amst) 11 (2012) 811-822.

[52] G. Minotti, P. Menna, E. Salvatorelli, G. Cairo, L. Gianni. Anthracyclines: molecular advances and pharmacologic developments in antitumor activity and cardiotoxicity, Pharmacol Rev 56 (2004) 185-229.

[53] F. Huang, A.V. Mazin. A small molecule inhibitor of human RAD51 potentiates breast cancer cell killing by therapeutic agents in mouse xenografts, PLoS One 9 (2014) e100993.

[54] F. Huang, A.V. Mazin. Targeting the homologous recombination pathway by small molecule modulators, Bioorg Med Chem Lett 24 (2014) 3006-3013.

[55] D.A. Alagpulinsa, S. Ayyadevara, R.J. Shmookler Reis. A Small-Molecule Inhibitor of RAD51 Reduces Homologous Recombination and Sensitizes Multiple Myeloma Cells to Doxorubicin, Front Oncol 4 (2014) 289.

[56] A. Alpi, P. Pasierbek, A. Gartner, J. Loidl. Genetic and cytological characterization of the recombination protein RAD-51 in Caenorhabditis elegans, Chromosoma 112 (2003) 6-16.

[57] C. Zakaria, H. Kassahun, X. Yang, J.C. Labbe, H. Nilsen, D. Ramotar. Caenorhabditis elegans APN-1 plays a vital role in maintaining genome stability, DNA Repair (Amst) 9 (2010) 169-176.

[58] Y. Popova, P. Thayumanavan, E. Lonati, M. Agrochao, J.M. Thevelein. Transport and signaling through the phosphate-binding site of the yeast Pho84 phosphate transceptor, Proc Natl Acad Sci U S A 107 (2010) 2890-2895.

[59] J. Schothorst, H.N. Kankipati, M. Conrad, D.R. Samyn, G. Van Zeebroeck, Y. Popova, M. RubioTexeira, B.L. Persson, J.M. Thevelein. Yeast nutrient transceptors provide novel insight in the functionality of membrane transporters, Curr Genet 59 (2013) 197-206.

[60] R.F. Gaber, K. Ottow, H.A. Andersen, M.C. Kielland-Brandt. Constitutive and hyperresponsive signaling by mutant forms of Saccharomyces cerevisiae amino acid sensor Ssy1, Eukaryot Cell 2 (2003) 922-929.

[61] L. Bianchi, A. Diez-Sampedro. A single amino acid change converts the sugar sensor SGLT3 into a sugar transporter, PLoS One 5 (2010) e10241.

[62] S. Tumusiime, C. Zhang, M.S. Overstreet, Z. Liu. Differential regulation of transcription factors Stp1 and Stp2 in the Ssy1-Ptr3-Ssy5 amino acid sensing pathway, J Biol Chem 286 (2011) 4620-4631.

(C2017 by the authors; licensee IAPC, Zagreb, Croatia. This article is an open-access article distributed under the terms and conditions of the Creative Commons Attribution license (http://creativecommons.org/licenses/by/3.0/) (cc) EY 